\title{
EXCITONIC SPECTRA OF $\mathrm{Cd}_{1-x} \mathrm{Fe}_{x}$ Te CRYSTALS
}

\author{
S.A. Permogorov, L.N. Tenishev \\ A.F. Ioffe Physical-Technical Institute, Russian Academy of Sciences \\ Politechnicheskaya 26, 194021 St.-Petersburg, Russia \\ AND T.P. SuRkova
}

Institute of Metal Physics, Russian Academy of Sciences, Ural Department Kovalevskaya 18, 620219 Ekaterinburg, GSP-170, Russia

\begin{abstract}
Strong modification of the optical spectra near the band-gap edge is observed in $\mathrm{Cd}_{1-x} \mathrm{Fe}_{x} \mathrm{Te}$ crystals as compared to the spectra of a pure compound. The evolution of the luminescence spectra at the increase in Fe concentration is represented by the change of radiative recombination channels from dominantly $\left(A^{0}, x\right)$ acceptor bound exciton emission in undoped CdTe to the free exciton luminescence in $\mathrm{Cd}_{1-x} \mathrm{Fe}_{x} \mathrm{Te}$ solid solutions.
\end{abstract}

PACS numbers: 78.20.Ci

The important part of physical properties of $\mathrm{Cd}_{1-x} \mathrm{Fe}_{x} \mathrm{Te}$ crystals depends on the relative location of the $\mathrm{Fe}^{2+}$ state energies with respect to the valence and conduction bands. It was supposed [1-3] that the donor level of isolated, substitutional $\mathrm{Fe}_{\mathrm{Cd}}$ is located in the forbidden gap at $E_{\mathrm{c}}=1.45 \mathrm{eV}$. With the increase in Fe concentration up to the alloy region the position of this state will correspond to the valence band edge of the alloy [4]. It seems interesting to compare emission processes in $\mathrm{Cd}_{1-x} \mathrm{Fe}_{x} \mathrm{Te}$ crystals with those in the pure CdTe.

The single crystals used in this study were grown by the Bridgman method at the Institute of Physics of the Polish Academy of Sciences. Photoluminescence (PL) and reflectivity measurements have been carried out in the entire range of the Fe solubility $0<x<0.04$ at temperatures $5-250 \mathrm{~K}$. A He-Ne laser was used as the excitation source. Spectra were recorded using a DFS-12 monochromator and a photomultiplier connected to a photon counting system.

The photoluminescence and reflectivity spectra are shown in Fig. 1. The composition of the samples and some characteristics of PL and reflectivity spectra are presented in Table. As it can be seen from Fig. 1, the PL spectra of $\mathrm{Cd}_{1-x} \mathrm{Fe}_{x} \mathrm{Te}$ crystals are qualitatively different from that for parent compound. For pure CdTe crystals the luminescence spectrum shows simultaneously several channels of radiative recombination, most important of which are free $\left(E_{x}=1.5964 \mathrm{eV}\right)$ and bound $\left(\left(D^{0}, x\right)=1.5931 \mathrm{eV},\left(A^{0}, x\right)=1.5899 \mathrm{eV}\right)$ exciton emission lines, the bands of donor-acceptor recombination and their LO phonon replicas [5]. In contrast, the emission spectrum of $\mathrm{Cd}_{1-x} \mathrm{Fe}_{x}$ Te with $3.5 \times 10^{19} \mathrm{~cm}^{-3}$ Fe concentration contains 
Free exciton positions $E_{\mathrm{FE}}$, the widths of free exciton reflection lines $\Delta=\left(E_{\mathrm{FE}}^{\min }-E_{\mathrm{FE}}^{\max }\right)$ and the luminescence quenching activation energies $E_{\text {act }}$ for studied samples. $E_{\mathrm{FE}}$ and $\Delta$ are taken at $5 \mathrm{~K}$.

\begin{tabular}{c|l|l|l}
\hline \hline Fe content & $E_{\mathrm{FE}}[\mathrm{eV}]$ & $\Delta[\mathrm{meV}]$ & $E_{\text {act }}[\mathrm{meV}]$ \\
\hline 0 & 1.5964 & 1.0 & 23.0 \\
$1 \times 10^{17} \mathrm{~cm}^{-3}$ & 1.5963 & 1.53 & 19.8 \\
$3 \times 10^{18} \mathrm{~cm}^{-3}$ & 1.5967 & 1.96 & 14.2 \\
$3.5 \times 10^{19} \mathrm{~cm}^{-3}$ & 1.5992 & 3.05 & 10.6 \\
$2 \%$ & 1.6157 & 7.4 & - \\
$4 \%$ & 1.6527 & 30.0 & -
\end{tabular}
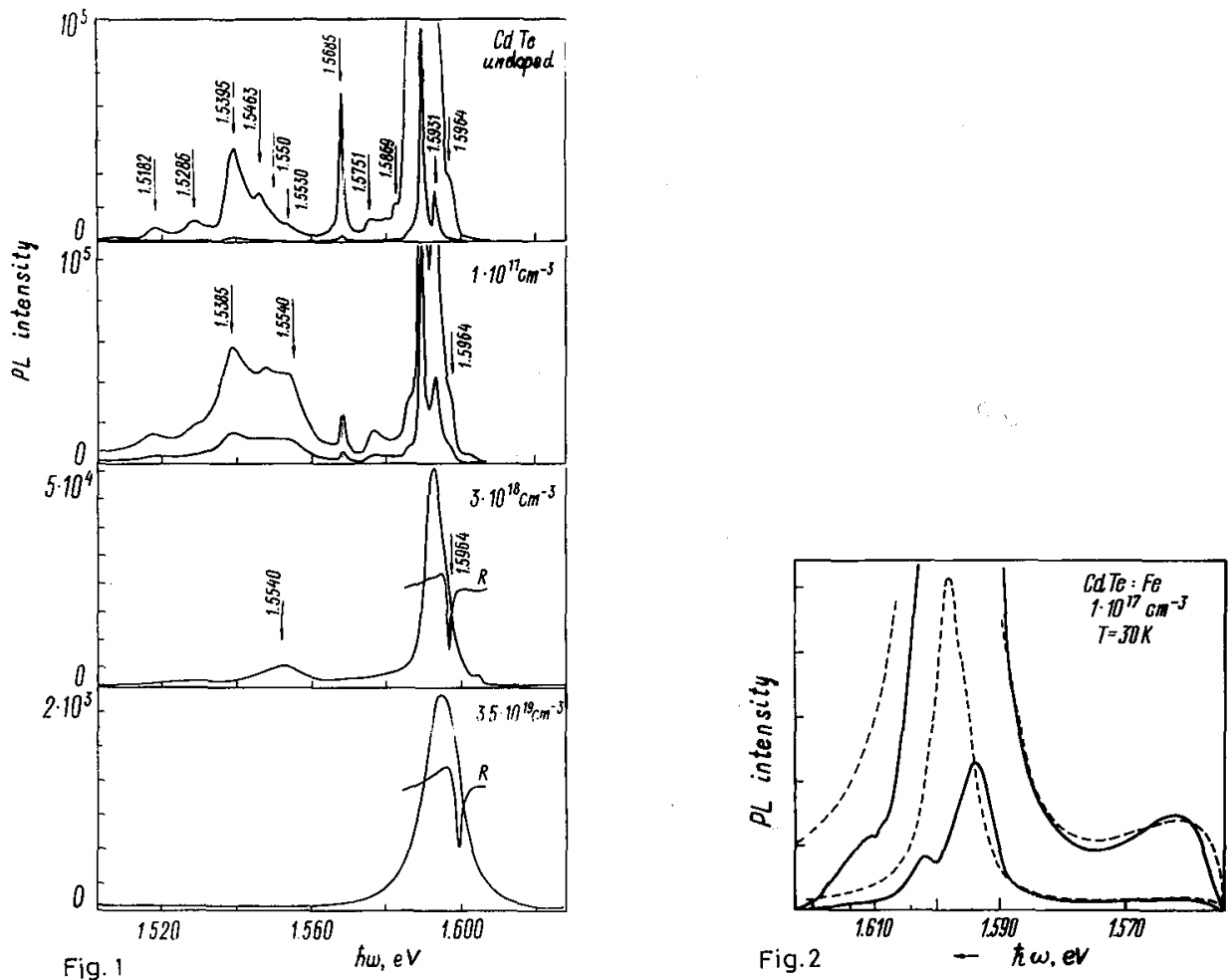

Fig. 1. PL spectra of $\mathrm{Cd}_{1-x} \mathrm{Fe}_{x} \mathrm{Te}$ crystals for different Fe concentration at $5 \mathrm{~K}$.

Fig. 2. The experimental PL spectrum of $\mathrm{Cd}_{1-x} \mathrm{Fe}_{x} \mathrm{Te}$ crystal (solid line) and the theoretical fit of the spectrum (dashed line) using Eq. (1) and the parameters $\varphi=0.007$, $\gamma=0.2, \Gamma=1.5 \mathrm{meV}$. 
only one broad but less intensive luminescence band. The maximum of the luminescence line is very close in position to the maximum of the exciton reflection line. We concluded that the evolution of the luminescence spectra at the increase in $\mathrm{Fe}$ concentration is connected with the change of the radiative recombination mechanism from dominantly $\left(A^{0}, x\right)$ acceptor bound exciton emission in undoped $\mathrm{CdTe}$ to the free exciton emission in $\mathrm{Cd}_{1-x} \mathrm{Fe}_{x} \mathrm{Te}$ solid solutions. The decrease in the activation energy $E_{\text {act }}$ from $23.0 \mathrm{meV}$ of $\left(A^{0}, x\right)$ bound exciton in pure CdTe to $10.6 \mathrm{meV}$ of free exciton in $\mathrm{Cd}_{1-x} \mathrm{Fe}_{x} \mathrm{Te}$ crystals confirms this interpretation (Table). Transition of Fe ions from $2+$ to $3+$ charge state is believed to be rcsponsible for the decrease in the neutral acceptor concentration. The dynamics of the temperature modification of the luminescence spectra is very similar to their evolution with the increase in Fe content. The excitonic character of recombination processes is confirmed also by the typical shape of $(A-\mathrm{LO})$ bands which are caused by exciton radiative recombination with simultaneous phonon emission. The example of such spectrum is shown in Fig. 2. The line shape of the exciton emission line $I(E)$ was fitted by the expression

$$
I(E)=\frac{\left[E^{\frac{1}{2}} \exp (-E / k T)+\varphi S(E)\right](1+\gamma E)}{\left(E-\omega_{\mathrm{LO}}\right)^{2}+\Gamma^{2}},
$$

where $E$ is the exciton kinetic energy, $S(E)$ - an additional non-cquilibrium contribution to the distribution function, $\varphi$ and $\gamma$ - parameters describing the relation of non-equilibrium/equilibrium distributions and processes with conservation/non-conservation of the wave vector, respectively. The expression (1) describes both the phonon-assisted ( $A-\mathrm{LO}$ ) band and the resonant exciton emission. The deviation of the theoretical curve from the experimental data in the region of the exciton resonance is caused by the strong reabsorption of exciton luminescence. The necessity to consider the non-equilibrium exciton distribution is due to the shortening of the exciton lifetime in Fe doped samples. The partial non-conservation of the wave vector in exciton recombination is caused by the exciton scattering on $\mathrm{Fe}$ impurities.

The authors thank A. Mycielski and W. Dobrowolski for supplying the $\mathrm{Cd}_{1-x} \mathrm{Fe}_{x} \mathrm{Te}$ crystals.

\section{References}

[1] A. Mycielski, J. Appl. Phys. 63, 3279 (1988).

[2] K. Lischka, G. Brunthauer, W. Jantsch, J. Cryst. Growth 72, 355 (1985).

[3] A.J. Szadkowski, J. Phys. Condens. Matter 2, 9853 (1990).

[4] B.A. Orłowski, B.J. Kowalski, A. Sarem, A. Mycielski, B. Velicky, V. Chab, Proc. 19th Int. Conf. Phys. Semicond., Vol. 2, Ed. W. Zawadzki, Institute of Physics, Polish Academy of Sciences, Warszawa 1988, p. 1267.

[5] R. Zanio, in: Semiconductors and Semimetals, Vol. 13, Academic Press, New York, London 1978. 